\title{
Overview of the Altair Lunar Lander Thermal Control System Design
}

\author{
Ryan A. Stephan \\ NASA Johnson Space Center
}

\begin{abstract}
NASA's Constellation Program has been developed to successfully return humans to the Lunar surface by 2020 . The Constellation Program includes several different project offices including Altair, which is the next generation Lunar Lander. The planned Altair missions are very different than the Lunar missions accomplished during the Apollo era. These differences have resulted in a significantly different thermal control system architecture.
\end{abstract}

The current paper will summarize the Altair mission architecture and the various operational phases. In addition, the derived thermal requirements will be presented. The paper will conclude with a brief description of the thermal control system designed to meet these unique and challenging thermal requirements.

\section{INTRODUCTION}

In response to President Bush's 2004 Vision for Space Exploration, NASA is planning a human return to the moon by 2020. In preparation for this mission, NASA has established the Constellation Program. Within the Constellation Program, there are several project offices. One of these projects includes the development of NASA's new Lunar lander vehicle. The overall mission architecture for this vehicle, Altair, is very similar to Apollo's architecture. This paper will provide the reader with a very brief overview of the Altair vehicle and mission architecture. As implied by the title, special consideration will be given to the vehicle's thermal control system. The thermal control system requirements, functionality, and hardware will be discussed.

\section{ALTAIR OVERVIEW}

The current Lunar mission architecture requires the use of two separate launch vehicles (Ares I and Ares V). In addition to these critical assets, a successful Lunar mission also requires an Earth Departure Stage (EDS), an Orion crewed vehicle, and the aforementioned Lunar lander. The larger of the two launch vehicles, Ares V, first lifts both the EDS and Altair into Low Earth Orbit (LEO). Shortly thereafter, the second launch vehicle, Ares I, launches Orion into LEO with four crewmembers inside the vehicle. Orion and the Altair/EDS stack rendezvous in LEO and the EDS propels the now integrated stack on a trajectory to the moon. The EDS is jettisoned immediately after the translunar injection burn.
The mission phase following the translunar injection burn is referred to as the translunar coast (TLC). The translunar coast duration is approximately three days. After Orion and Altair arrive at the moon, Altair is used to insert the vehicles into a $100 \mathrm{~km}$, circular Low Lunar Orbit (LLO). The vehicles spend approximately 24 hours in LLO before Altair separates from Orion and begins its descent to the Lunar surface. The entire descent phase takes approximately four hours to complete.

Once on the surface of the moon, the Lunar surface phase is started and lasts for approximately seven days. Throughout this time, the crew will perform up to seven extravehicular activities (EVAs). Each EVA includes two crewmembers for a total of eight hours. In total, approximately 112 man-hours will be spent outside of the pressurized cabin. Once the seven days have elapsed, the ascent module separates from the descent module and the airlock. The ascent module then carries the four crewmembers back to the awaiting Orion capsule where the vehicles again rendezvous in LLO. Once aboard Orion, the Altair ascent module is jettisoned back to the Lunar surface. Orion's service module pushes the crew back to Earth where the Orion crew module and the four crewmembers land safely in the ocean ${ }^{1}$.

As alluded to in the preceding paragraphs, Altair consists of three functional components as shown in Figure 1. The largest and most massive functional component is the Descent Module (DM). The DM is an unpressurized module with composite struts and includes the main propulsion system required to perform the Lunar orbit insertion burn, Lunar descent, and landing on the Lunar surface. The main propulsion system is composed of eight massive propulsion tanks. Of these tanks, four contain liquid hydrogen while the remaining four tanks are filled with liquid oxygen. The propellant within these tanks is required to fuel the engine throughout the mission. The descent module is powered using fuel cells. 


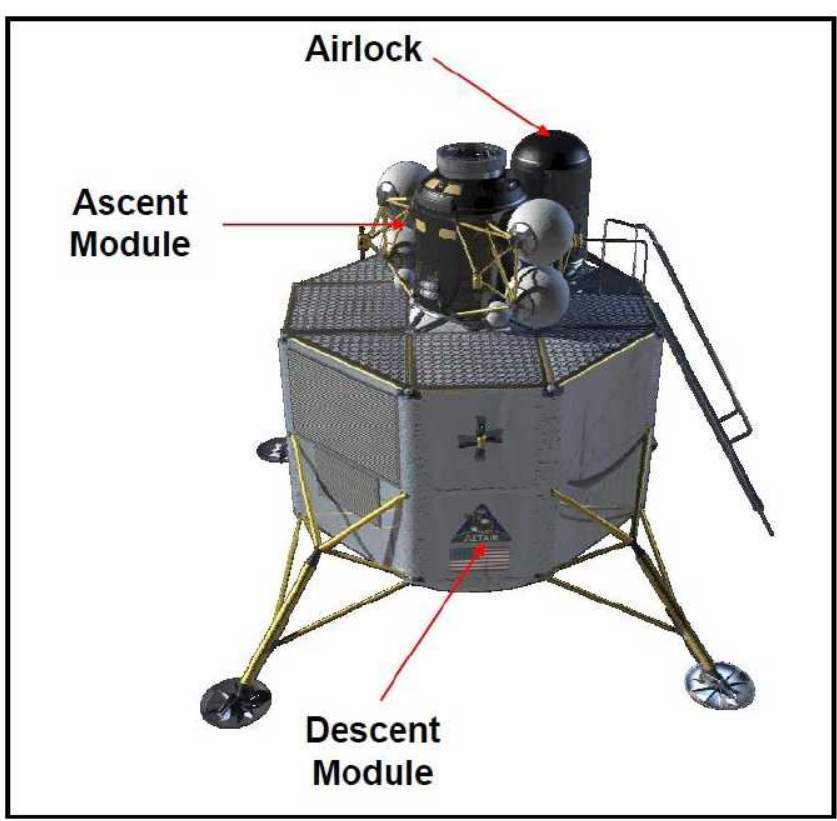

Figure 1. Altair Lunar lander showing the three functional components (Airlock, Ascent Module, and Descent Module.

The two smaller structures shown in Figure 1 are referred to as the Ascent Module (AM) and the AirLock (AL). As indicated by the name, the AM is used to return the crew to the waiting Orion after the Lunar surface stay. The overwhelming majority of the avionics and a small ascent engine are included in the AM design. The AM also serves as the habitable volume for the four crewmembers during descent, ascent, and the seven-day Lunar surface stay. The AM is nominally pressurized to 10.2 psia while docked with Orion and 8.3 psia when separated from Orion. During ascent, the AM is powered using on-board batteries. The $\mathrm{AL}$ is attached to the AM through a docking tunnel and is maintained at the same pressure as the AM throughout the entire mission except when it is depressurized for ingress and egress. The AL is used to ingress and egress the vehicle during extravehicular activities and is designed to accommodate two crewmembers per ingress/egress. The $\mathrm{AL}$ is depressurized and repressurized several times during Lunar surface operations. While the AM performs the Lunar ascent, both the $\mathrm{DM}$ and the $\mathrm{AL}$ remain on the Lunar surface. After the AM rendezvous with Orion, the $\mathrm{AM}$ is jettisoned and returned to the Lunar surface.

As mentioned above, Altair is designed to take a crew of four to the Lunar surface and support surface operations for up to seven days. These mission requirements far exceed the Apollo capability of two crewmembers for a total of three days on the Lunar surface. In addition to these differences, another major difference is that Altair is required to perform the Lunar orbit insertion burn. On Apollo, the command/service module was responsible for performing the Lunar orbit insertion burn. However, because Orion is being optimized for earlier LEO missions, the Lunar orbit insertion burn will be performed by Altair, which resulted in a much heavier landing vehicle as compared to the Apollo design.

\section{THERMAL CONTROL SYSTEM OVERVIEW}

From the simplest satellites to the most complex manned space vehicles, some degree of thermal control is required for all spacecraft. In its most basic form, thermal control is the maintenance of all vehicle surfaces and components within an appropriate temperature range. For satellites, this requires that the thermal control system must maintain all of the equipment within its operating temperature range. Similar to satellites, the thermal control system for manned space vehicles must also maintain all of the equipment within its operating temperature. In addition to component level temperature maintenance, the manned spacecraft's thermal control system must also safely maintain the internal cabin temperature within the appropriate temperature requirements to ensure crew survivability and comfort.

An effective thermal control system must provide three basic functions to the vehicle design. The three functions are heat acquisition, heat transport, and heat rejection. Heat acquisition is the process of transferring thermal energy from the heat source to the thermal control system. The second critical function of a thermal control system is heat transport and involves the movement of thermal energy from one region to another. The third and final function is heat rejection and is the process of rejecting the excess thermal energy acquired by the thermal control system to the external environment.

\section{ALTAIR THERMAL CONTROL SYSTEM HEAT REJECTION REQUIREMENTS}

The first step in designing Altair's thermal control system is to understand the derived thermal system heat rejection requirements. The system requirements are developed using knowledge of the vehicle's power requirements, the thermal environment, and the efficiency of the power generation system. For Altair, the vehicle power is generated using either fuel cells or batteries depending on the mission phase. The vehicle's heat rejection requirement varies throughout the Lunar mission and is shown in Figure 2.

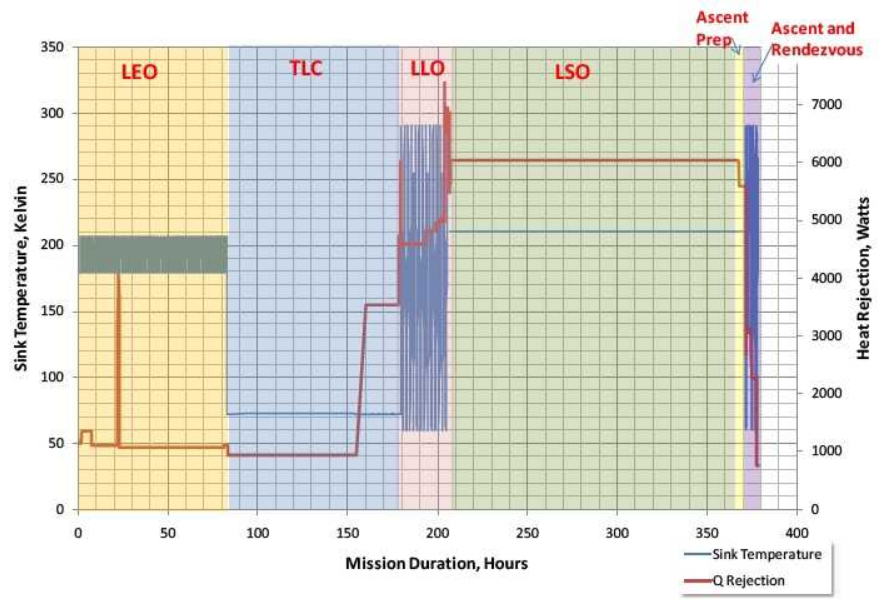

Figure 2. Altair heat rejection requirement from Low Earth Orbit (LEO) through the completion of Ascent Module disposal.

In the preceding figure, the heat rejection requirement is located along the right abscissa and the Mission Elapsed Time (MET) is shown along the ordinate. The abscissa shown on the left side of the plot represents the radiator sink temperature 
assuming optical properties representative of silverized Teflon tape $(\alpha=0.10, \varepsilon=0.85)$. The instantaneous sink temperature is calculated using the following equation:

$T_{s}=\left[\frac{\frac{\alpha}{\varepsilon} q_{\text {solar }}^{\prime \prime}+q_{I R}^{\prime \prime}}{\sigma}\right]^{1 / 4}$

Where:

$\mathrm{T}_{\mathrm{s}}$ represents the radiative sink temperature (Kelvin)

$\alpha$ represents the radiator solar absorptivity (unitless)

$\varepsilon$ represents the radiator infrared emissivity (unitless)

q" solar represents the incident solar flux $\left(\mathrm{W} / \mathrm{m}^{2}\right)$

q" ${ }_{I R}$ represents the incident infrared flux $\left(\mathrm{W} / \mathrm{m}^{2}\right)$

$\sigma$ represents the Stefan-Boltzmann constant $\left(\mathrm{W} / \mathrm{m}^{2}-\mathrm{K}^{4}\right)$

The plot is color-coded by the location in the mission profile. Starting from MET of zero, the gold region represents the period of time that the vehicle is located in Low Earth Orbit (LEO). During LEO, the heat rejection requirement is close to 1000 Watts for the majority of the mission profile. However, there is a small period of time that the heat rejection requirement spikes up to just over 4000 Watts. This spike in the vehicle's heat rejection requirement corresponds to the time when the vehicle is checking out several systems including the fuel cells. The corresponding sink temperature shown in this figure ranges between 180 and 210 Kelvin.

| The following purple region is the portion of the mission when the vehicle is in TransLunar Coast (TLC). During this mission phase the heat rejection requirement is less than 1000 Watts and then increases to approximately 3400 Watts as the vehicle prepares for the Lunar Orbit Insertion (LOI) burn. As shown in this figure, TLC is an extremely cold thermal environment. The radiative sink temperature during this mission phase is less than 80 Kelvin for the duration of the phase.

Unlike the previous mission phases, the sink temperature in Low Lunar Orbit varies significantly throughout the phase. As shown in Figure 2, the radiative sink temperature for LLO varies between 60 Kelvin when the sun is shadowed by the Moon itself. However, when the vehicle is located directly between the sun and the Lunar surface, the sink temperature is approximately 290 Kelvin. This wide variation in sink temperature results in a significant design challenge that will be discussed later in this document. As is the case with the sink temperature, the heat rejection requirement also varies throughout this mission phase from a minimum value of 4550 Watts to a maximum requirement of 7380 Watts. The timeweighted average (which was used for subsequent thermal analysis) heat rejection requirement during LLO is approximately 4800 Watts.

The next mission phase, color coded green in Figure 2, is the Lunar Surface Operations (LSO). For the majority of LSO, the heat rejection requirement is a constant 6040 Watts. However, near the end of this mission phase, the heat rejection requirement does drop to approximately 5600 Watts while the vehicle is being prepared for Lunar ascent. During the seven minute ascent phase, the heat rejection requirement is 2679 Watts. The remaining mission phases (rendezvous with
Orion, docked operations with Orion, and preparation for disposal) occur in Low Lunar Orbit. During these mission phases the heat rejection requirement varies between 970 Watts and 3100 Watts. The thermal control system must continue to function throughout the ascent module disposal. During this period of time, the ascent module heat rejection requirement is only 750 Watts.

\section{ALTAIR THERMAL CONTROL SYSTEM ARCHITECTURE}

Thermal control systems are typically designed for the highest heat rejection requirement in the warmest continuous thermal environment. Figure 2 clearly shows that this scenario occurs during Lunar surface operations. As mentioned above, the heat rejection requirement during LSO is 6040 Watts and the corresponding radiator sink temperature is approximately 210 Kelvin. The Altair thermal control system schematic developed to meet the aforementioned system requirements is shown in Figure 3. 


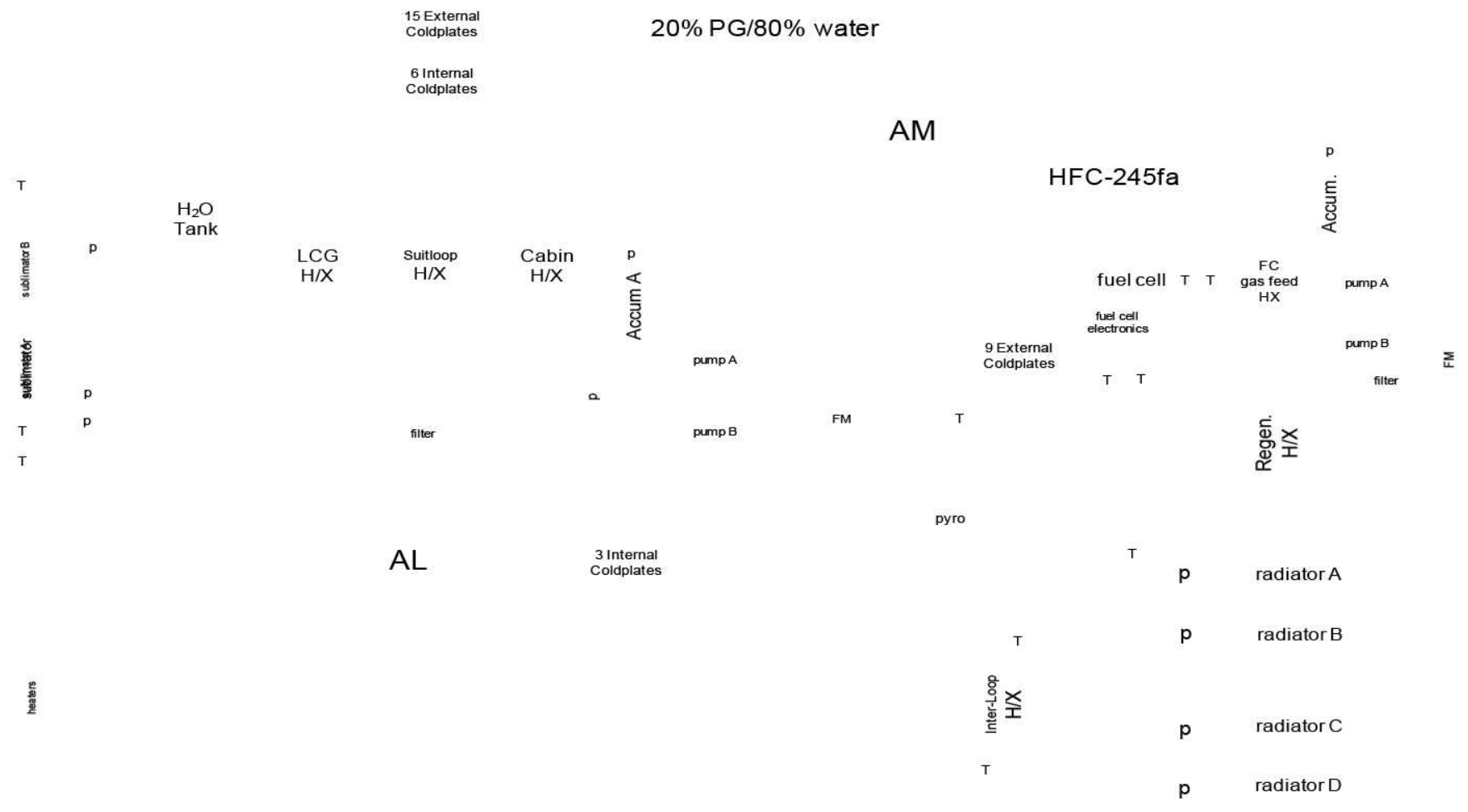

Figure 3. Altair thermal control system schematic.

Altair's thermal control system accomplishes the heat acquisition function using various coldplates and heat exchangers as shown in the preceding figure. There are two different types of heat exchangers included in the system design. One of these heat exchanger types is an air/liquid heat exchanger. As the name implies this type of heat exchanger is used to transfer thermal energy from an air loop to a liquid cooling loop. The thermal control system design includes two air/liquid heat exchangers. The primary air/liquid heat exchanger, which is labeled "Cabin HX" in Figure 3, transfers energy from the air in the ascent module to the thermal control system. The majority of this thermal load is from the crew metabolic load, but the thermal load also includes airborne energy from the various avionics located in the airlock and ascent module. The second air/liquid heat exchanger is used to acquire energy from a suited crewmember. This heat exchanger is only used during mission phases when the crewmembers are in their spacesuit. The second type of heat exchanger is a liquid/liquid heat exchanger and this hardware acquires energy from one liquid loop and transfers it to a second liquid loop. There are a total of three liquid/liquid type heat exchangers. The first is the Liquid Cooling Garment (LCG) heat exchanger and its purpose is to acquire energy from the crew's LCG and transfer it to the thermal control system. As before, this heat exchanger is only used when the crewmembers are in their spacesuits. The second liquid/liquid heat exchanger is the inter-loop heat exchanger. This hardware is used to transfer energy from the internal pumped fluid loop to the external pumped fluid loop. The third, and final, liquid/liquid heat exchanger is the regenerative heat exchanger ${ }^{2}$ and it is used to maintain the system setpoint throughout the mission phases where the radiators are being used.
There are a total of 33 coldplates included in the Altair thermal control system. The overwhelming majority (21 of 33 ) are located on the vehicle's ascent module. The coldplates are required to acquire excess thermal energy from various avionics components while maintaining these devices within their acceptable temperature limits. The heat-generating components include computers, inertial measurement units, computer monitors, etc.

The second critical function of a thermal control system is heat transport. For the Altair system design, the heat transport function is accomplished using a two-loop system architecture. A two-loop architecture was required due to concerns about the fluid inside the radiator freezing during translunar coast. The system heat rejection requirement is very low during this mission phase. This fact combined with TLC being an extremely cold thermal environment leads to concerns regarding fluid freeze. Unfortunately, fluids with low enough freeze temperatures (less than 170 Kelvin) are toxic, which is a concern in the event of an inadvertent fluid leak in the pressurized module. Therefore, safety concerns lead to the selection of a non-toxic fluid for any loop inside the ascent module or airlock.

The resulting internal pumped fluid loop (depicted as the "red" lines in Figure 3) uses a mixture of propylene glycol and water as the working fluid. In part, this particular fluid formulation was chosen to ensure commonality with the Orion system design $^{3}$. There is a strong desire within the Constellation Program for commonality across the elements. This formulation provides a small level of freeze protection and is said to be compatible with aluminum. This loop gathers thermal energy from the various heat exchangers and coldplates located in both the ascent module and the airlock. Another unique aspect of the internal loop design is that it is 
used to provide condensation control for the vehicle. This function is accomplished by incorporating "omega loops" (shown as the five omega shaped figures in Figure 3). The omega loops are simply fluid loops that are thermally coupled to areas of the spacecraft that are particularly susceptible to condensation (hatches, windows, etc.).

The internal loop is driven by a centrifugal pump and the system flowrate is approximately $250 \mathrm{lb} / \mathrm{hr}$. Due to the criticality of the system (failure of the system would lead to loss of crew), a parallel redundant pump is included in the design. The secondary pump serves as a "cold back-up" because it would only be activated if the primary pump failed at any time during the mission. The energy acquired by the internal loop is transferred to the external loop through the inter-loop heat exchanger.

The external loop is also driven by a centrifugal pump, which is backed up by an identical inactive pump. The baseline working fluid for the external loop is HFC-245fa. This fluid was chosen due to its low freezing temperature and desirable mechanical properties. HFC-245fa is a hydro-fluoro-carbon and was developed as a replacement to the ozone-depleting chlorofluorocarbon. The flowrate for the HFC-245fa is a constant $1200 \mathrm{lb} / \mathrm{hr}$. Once the external loop acquires energy from the internal loop, it continues to service several coldplates and also provides cooling for the fuel cells while they are operating. After acquiring energy from the fuel cells, the loop is also used to condition the fuel cell reactants prior to entering the power-generating device. The entirety of the acquired energy is then transported to the radiator panels where it is rejected to space.

The Altair thermal control system relies on two separate hardware systems to achieve the heat rejection function of the system. For the majority of the mission, heat rejection is accomplished using four body-mounted radiator panels located on the descent module. The four radiator panels are located $90^{\circ}$ apart from one another and are insulated on the backside (side pointing towards the cryogenic tanks). The radiator panels were sized for Lunar surface operations and the total surface area is approximately $24 \mathrm{~m}^{2}$. As each of the panels is identical, each panel is 1.8 meters wide by 3.4 meters long.

The radiator panels are sufficiently sized to reject the full vehicle heat load throughout all of the mission phases up to low Lunar orbit. There are several instances during this mission phase where the radiator's thermal environment is too warm for the radiators to reject the full vehicle heat load while maintaining the system setpoint. The warm thermal environment is due to the high infrared backload incident upon the vehicle's radiator while situated above the Lunar surface's sub-solar point. The sub-solar point is the point on the Lunar surface directly aligned with the sun. Figure 4 shows the spatial variation of temperature on the Lunar surface.

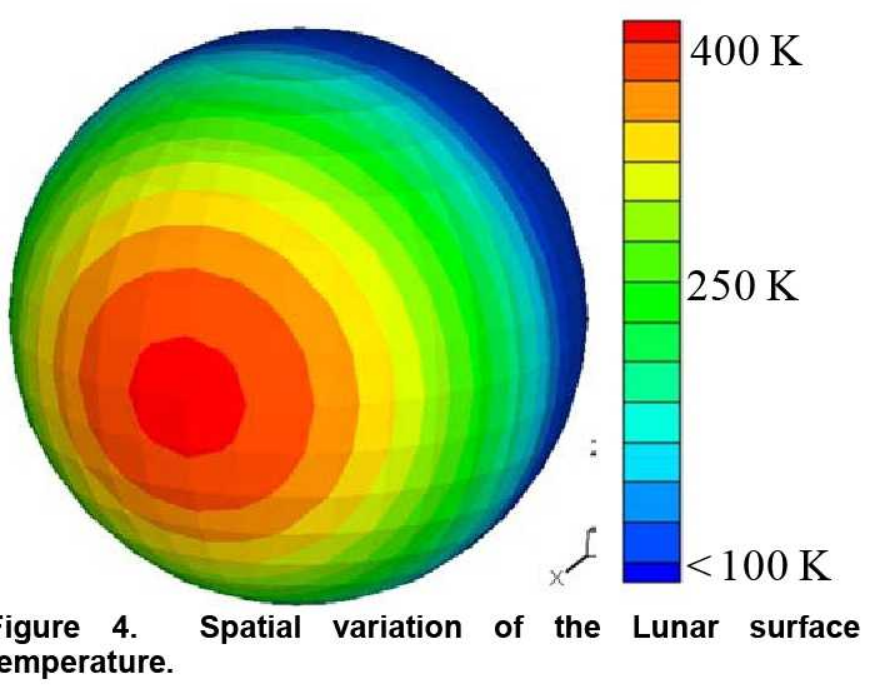

As shown in the preceding figure, the Lunar surface temperature at the sub-solar point reaches temperatures approaching 400 Kelvin. On the other hand, the surface temperature on the cold side of the moon is less than 100 Kelvin. These wide variations in surface temperature result in a variable incident infrared heat flux on an orbiting vehicle.

Using knowledge of the varying incident IR and solar loads, an instantaneous sink temperature can be calculated for a 100 kilometer, circular orbit using Equation 1. The corresponding instantaneous (thermal mass of the system was not considered for this analysis) radiator capability for these orbital parameters is shown in Figure 5.

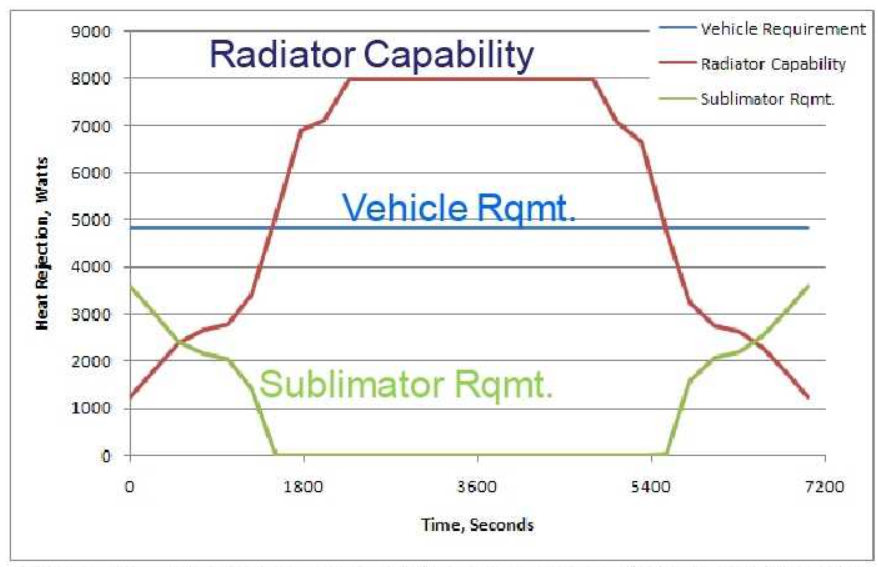

Figure 5. Radiator capability and associated sublimator requirement for a circular $100 \mathrm{~km}$ Lunar orbit.

In the preceding figure, the vertical axis represents the heat rejection, while the horizontal axis is the duration of the low Lunar orbit. Time zero corresponds to the time when the vehicle is situated immediately above the sub-solar point. At this time, the radiator capability (shown in "red") is quite low given the relatively warm sink temperature associated with this orbital position. However, as the vehicle orbits the moon, the sink temperature decreases and the associated radiator capability increases to almost 8000 Watts. As was discussed above, the time-weighted average heat rejection requirement during LLO is approximately 4800 Watts (shown in "blue"). In studying Figure 5, it is apparent that there are several instances during which the radiator is not capable of rejecting the full vehicle heat load. During these instances, a 
Supplemental Heat Rejection Device (SHReD) is required. The requirement for the SHReD can be obtained by simply subtracting the radiator capability from the vehicle heat rejection requirement. The resulting SHReD requirement is shown as the "green" curve in Figure 5. During the periods requiring supplemental heat rejection a sublimator is used to reject the excess energy not rejected using the radiator panels. A sublimator was chosen as the SHReD because this type of hardware was already included in the system design for Lunar ascent. The Altair thermal control system includes two identical sublimators. The second sublimator serves as a cold back-up to be used in the event that the primary sublimator fails. A sublimator is a type of evaporator that requires a consumable feedwater. The consumable feedwater is supplied to the sublimator where it freezes when exposed to space vacuum. The now frozen feedwater layer sublimates to space when energy is applied. The mass of feedwater required to perform this supplemental heat rejection function for a single orbit was calculated using the following equation:

$m=\frac{E_{S H R e D}}{h_{f g}}=\frac{\int_{0}^{t_{f}} Q_{S H R e D}(t) d t}{h_{f g}}$

Where:

$\mathrm{m}$ represents the mass of the consumed feedwater $(\mathrm{kg})$

$E_{\text {SHReD }}$ represents the amount of energy required to be

removed via sublimation (Joules)

$t_{f}$ represents the duration of a Lunar orbit (seconds)

$\mathrm{Q}_{\text {SHReD }}(\mathrm{t})$ represents the time-varying heat rejection

requirement for the $\mathrm{SHReD}$, which is shown as the "green"

curve in Figure 5 (Watts)

$\mathrm{h}_{\mathrm{fg}}$ represents the heat of vaporization for the consumable

feedwater $(\mathrm{J} / \mathrm{kg})$

The duration of the low Lunar orbit mission phase is approximately 26 hours as shown in Figure 2. During this period the sublimator consumes approximately $36 \mathrm{~kg}$ of feedwater to meet the vehicle's heat rejection requirements. It is important to note that the preceding analysis assumed a sublimator utilization of $100 \%$. There is a concern that the true utilization may be less than $100 \%$ when the sublimator is used in this cyclical fashion ${ }^{4}$.

During Lunar descent, a sublimator is used as the only means of heat rejection. This assumption was made due to uncertainty surrounding the thermal environment during Lunar descent. It is plausible that the thermal environment during descent is too warm for the radiators to reject the full vehicle heat load. Therefore, in an effort to be conservative, the system was designed to only use a sublimator during this mission phase. A total of $11.8 \mathrm{~kg}$ of water is consumed during Lunar descent. As mentioned above, the entire thermal control system, including the radiators, were sized for Lunar surface operations. Therefore, there is not a need for the sublimator during Lunar surface operations.

Because the airlock and the descent module are left on the Lunar surface during ascent, there are two pyrotechnic valves included in the thermal control system design. These pyrotechnic valves are shown as red x's in Figure 5. Just prior to ascent, several isolation valves are configured to isolate the internal thermal control system for ascent. These valves are used to bypass the airlock and only direct the fluid flow through the ascent module. After configuring these valves, the pyrotechnic valves are used to sever the internal thermal pumped fluid loop lines. Because the radiator panels remain on the Lunar surface with the descent module, the sublimator provides the heat rejection function throughout Lunar ascent, Orion rendezvous, docked operations, and vehicle disposal. Figure 6 shows the required sublimator feedwater mass for each of the mission phases where a sublimator is used as either supplemental heat rejection or primary heat rejection.

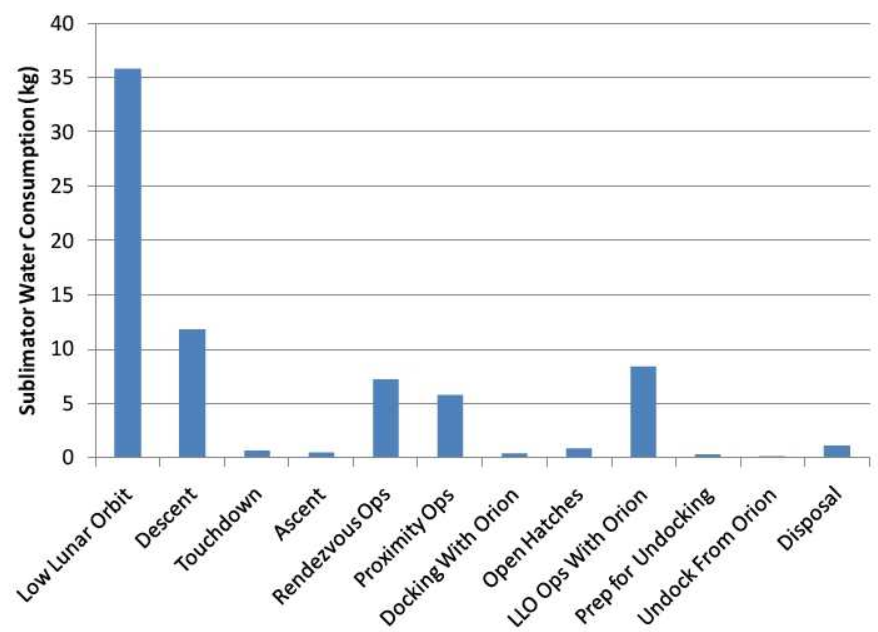

Figure 6. Mass of sublimator feedwater used throughout Altair's mission profile.

A total of $73 \mathrm{~kg}$ of water is required to meet the vehicle's heat rejection needs during the 16-day mission. The overwhelming majority of that water is consumed during low Lunar orbit where the sublimator serves as the supplemental heat rejection device. The other mission phases that require a large amount of sublimator water are Lunar descent, rendezvous and proximity operations, and LLO operations with Orion.

\section{CONCLUSIONS}

This paper has provided a brief overview of the thermal control system design for the Altair Lunar lander. The system was designed to effectively operate through a wide range of thermal environments and was designed to meet the derived heat rejection requirements for Altair. These transient requirements were generated with knowledge of the vehicle's power requirements, thermal environments, and crew metabolic load requirements. The heat acquisition function is accomplished using several heat exchangers and coldplates. A two-loop architecture using both a mixture of propylene glycol and water as well as HFC-245fa is used to transport the acquired thermal energy to the heat rejection system. The heat rejection function is accomplished using four body-mounted radiators and, when necessary, a sublimator.

\section{ACKNOWLEDGEMENTS}

The author would like to thank Dr. Eugene Ungar and Greg Schunk for their insight and contributions to the thermal control system design. In addition, the author would like to thank his division management and Altair project office personnel for giving him the opportunity to work on NASA's next generation Lunar lander. He would also like to thank Dr. 
Tom Leimkuehler for being there to provide guidance and to serve as a "sounding board" for various technical issues that have come up throughout this design.

\section{REFERENCES}

1. Polsgrove, T., Button, R., Linne, D. "Altair Lunar Lander Consumables Management." AIAA SPACE 2009 Conference \& Exposition. September 2009.

2. Ungar, E. K. "Spacecraft Radiator Freeze Protection Using a Regenerative Heat Exchanger with Bypass Setpoint Temperature Control." SAE ICES Paper 08ICES-2170. July 2008.

3. Lewis, J., Barido, R., Carrasquillo, R., Cross, C., Rains, E., Tuan, G. "Crew Exploration Vehicle Environmental Control and Life Support Development Status." SAE ICES Paper 09ICES-2457. July 2008.

4. Leimkuehler, T. O., Sheth, R., Stephan, R. A. "Investigation of Transient Sublimator Performance." SAE ICES Paper 09ICES-0284. July 2009. 